\title{
Absolutely Continuous Spectrum and Scattering in the Surface Maryland Model
}

\author{
François Bentosela Philippe Briet Leonid Pastur
}

\begin{abstract}
We study the discrete Schrödinger operator $H$ in $\mathbf{Z}^{d}$ with the surface quasi periodic potential $V(x)=g \delta\left(x_{1}\right) \tan \pi\left(\alpha \cdot x_{2}+\omega\right)$, where $x=\left(x_{1}, x_{2}\right)$, $x_{1} \in \mathbf{Z}^{d_{1}}, x_{2} \in \mathbf{Z}^{d_{2}}, \alpha \in \mathbf{R}^{d_{2}}, \omega \in[0,1)$. We first discuss a proof of the pure absolute continuity of the spectrum of $H$ on the interval $[-d, d]$ (the spectrum of the discrete Laplacian) in the case where the components of $\alpha$ are rationally independent. Then we show that in this case the generalized eigenfunctions have the form of the "volume" waves, i.e. of the sum of the incident plane wave and reflected from the hyper-plane $\mathbf{Z}^{d_{1}}$ waves, the form that is well known in the scattering theory for decaying potential. These eigenfunctions are orthogonal, complete and verify a natural analogue of the Lippmann-Schwinger equation. We find the wave operators and the scattering matrix in this case. We discuss also the case of rational $\alpha=p / q$ 's, $p, q \in \mathbb{N}$ for $d_{1}=d_{2}=1$, i.e. of a periodic surface potential. In this case besides the volume waves there are also the surface waves, whose amplitude decays exponentially as $\left|x_{1}\right| \rightarrow \infty$. For large $q$ corresponding part of the absolutely continuos spectrum consists of $q$ exponentially narrow bands, lying all except one outside the interval $[-2,2]$, and converging in a natural sense as $q \rightarrow \infty$ to the dense point spectrum found before in [13] for the irrational Diophantine $\alpha$ 's.
\end{abstract}

\section{Introduction}

This paper is in the framework of problems discussed in [10] and later in [13], [4][9]. The problems concern spectral and related properties of differential and finitedifference operators either defined on the half-space by mean of random, almost periodic or periodic boundary condition or having the same type of coefficients supported on hyper-planes $\mathbb{R}^{d_{2}}$ or $\mathbb{Z}^{d_{2}}$ of the spaces $\mathbb{R}^{d}$ or $\mathbb{Z}^{d}$. In particular in [13] the discrete Schrödinger operator $H$, acting in $l^{2}\left(\mathbb{Z}^{d}\right)$ and having the "surface" potential

$$
V(\mathbf{x})=g \delta\left(x_{1}\right) \tan \pi\left(\alpha \cdot x_{2}+\omega\right), \mathbf{x}=\left(x_{1}, x_{2}\right), x_{1} \in \mathbb{Z}^{d_{1}}, x_{2} \in \mathbb{Z}^{d_{2}}, d_{1}+d_{2}=d,
$$


was considered. It was shown that for any $g \neq 0, \omega \in[0,1)$, and for $\alpha \in \mathbb{R}^{d_{2}}$ verifying the Diophantine condition:

$$
\left|\alpha \cdot x_{2}-m\right| \geq \text { const. } /\left|x_{2}\right|^{d_{2}+\epsilon}, \epsilon>0, x_{2} \in \mathbb{Z}^{d_{2}} \backslash\{0\}, m \in \mathbb{Z},
$$

the spectrum of $H=H_{0}+V$, lying outside the spectrum $[-d, d]$ of the discrete Laplacian $H_{0}$, is pure point, dense, of multiplicity one and the respective eigenfunctions decay exponentially at infinity.

The "volume" version of this operator corresponding, to the case $d_{1}=0$, has been studied in $[2,19,15]$. It can be viewed as a simple explicitly soluble model of a quasi periodic discrete Schrödinger operator in the strong localization regime. The operator has a complete system of exponentially decaying eigenfunctions, corresponding to the pure point dense spectrum of multiplicity one occupying the whole spectral axis.

In this paper, we study the absolutely continuous spectrum of $H$, i.e. the part of the spectrum complementary to that considered in [13]. This part of the spectrum was already studied in [6] in the context of the boundary value problem defined by (1.1) with $x_{1}=0$ (see formula (3.2) below). It was proven that if the vector $\alpha \in \mathbb{R}^{d_{2}}$ has rationally independent components, then the lying in $[-d, d]$ spectrum of the boundary value problem is purely absolutely continuous, and that the suitably defined wave operators for the pair exist and are complete. Besides, it was proven in [9] that in this case the surfaces states (see [10, 9] for definitions) are absent.

We first present a rather transparent proofs of the absolute continuity of the part $\sigma(H) \cap[-d, d]$ of the spectrum $\sigma(H)$ of $H$ for arbitrary $d_{1}, d_{2} \geq 1$ and of the completeness of the wave operators $\Omega_{ \pm}$for the pair $\left(H, H_{0}\right)$. Then we find a complete and orthonormal system of generalized eigenfunctions $\Psi_{ \pm}(\mathbf{x}, \mathbf{k}), \mathbf{x} \in \mathbb{Z}^{d}, \mathbf{k} \in \mathbb{T}^{d}$ (see formula (3.6)), having a "Sommerfeld-like" form of the sum of the incident plane wave of the unit amplitude and of the reflected waves and we prove that $\Psi_{ \pm}(\mathbf{x}, \mathbf{k})$ are the kernels of the wave operators $\Omega_{ \pm}$.

We report also certain results on the periodic two-dimensional case $d_{1}=d_{2}=1$, i.e. for $\alpha=p / q, p, q \in \mathbb{Z}^{1}$. In particular, we find the surface states and study their certain properties.

\section{Generalities}

Recall that we are studying the self-adjoint operator $H$, acting on $l^{2}\left(\mathbb{Z}^{d}\right)$ and having the form

$$
H=H_{0}+V
$$

where

$$
\left(H_{0} \Psi\right)(\mathbf{x})=-1 / 2 \sum_{|\mathbf{x}-\mathbf{y}|=1} \Psi(\mathbf{y}):=-\frac{1}{2} \Delta \Psi(\mathbf{y})
$$

is a discrete Laplacian, and $V$ is defined in (1.1). We will use now an analogue of the Cayley transform introduced in [2] in the case of the "volume" potential (1.1) $\left(d_{1}=0\right)$ and in [13] in the "surface" case $\left(d_{1}=1\right)$, in both cases to study the pure point spectrum for the Diophantine $\alpha^{\prime} s$ (see (1.2)). 
To put the subsequent simple argument in a general context, we rewrite the potential (1.1) as follows:

$$
V(\mathbf{x})=g \tan \pi\left(\alpha \cdot x_{2}+\omega\right) \chi_{S}(\mathbf{x}), \mathbf{x}=\left(x_{1}, x_{2}\right), x_{1} \in \mathbb{Z}^{d_{1}}, x_{2} \in \mathbb{Z}^{d_{2}},
$$

where $\chi_{S}$ is the indicator of the subspace $S=\mathbb{Z}^{d_{2}}$, and we assume that $g>0$ (the case $g<0$ can be treated analogously). $\chi_{S}$ can be regarded as the matrix of the orthogonal projection onto the subspace of $l^{2}\left(\mathbb{Z}^{d}\right)$ of functions having the support in $S$. Denote respective projection $P_{S}$ and write the potential (2.3) as follows:

$$
V=P_{S} v P_{S}, \quad v\left(x_{2}\right)=g \tan \pi\left(\alpha \cdot x_{2}+\omega\right) \chi_{s}(x) .
$$

Recall the well known formulas for the resolvent $G(z)=(H-z)^{-1}$ of a pair of selfadjoint operators $\left(H, H_{0}\right)$ :

$$
G(z)=G_{0}(z)-G_{0}(z) T(z) G_{0}(z)
$$

where

$$
G_{0}(z)=\left(H_{0}-z\right)^{-1}, \quad T(z)=V-V T(z) G_{0}(z) .
$$

In our case of the potential of the form (2.3) the operator $T(z)$ :

$$
T(z)=P_{S} t(z) P_{S}
$$

where the operator

$$
t(z)=v\left(1+P_{S} G_{0}(z) P_{S} v\right)^{-1}=\left(v^{-1}+P_{S} G_{0}(z) P_{S}\right)^{-1}
$$

acts on $P_{S} l^{2}\left(\mathbb{Z}^{d}\right)=l^{2}(S)$. Denote by $u$ the unitary operator on $l^{2}(S)$ defined as:

$$
(u \psi)\left(x_{2}\right)=e^{-2 i \pi \alpha \cdot x_{2}} \psi\left(x_{2}\right), \quad x_{2} \in S .
$$

We will also need the Fourier transform which we define as follows. For any function (any sequence) $\Phi \in l^{2}\left(\mathbb{Z}^{\nu}\right), \nu \geq 1$, we set

$$
\hat{\Phi}(\mathbf{k})=\sum_{\mathbf{x} \in \mathbb{Z}^{\nu}} e^{-2 i \pi \mathbf{x} \cdot \mathbf{k}} \Phi(\mathbf{x}), \quad \mathbf{k} \in \mathbb{T}^{\nu}
$$

Then we have

$$
\Phi(\mathbf{x})=\int_{\mathbb{T}^{\nu}} d \mathbf{k} e^{2 i \pi \mathbf{x} \cdot \mathbf{k}} \hat{\Phi}(\mathbf{k}), \quad x \in \mathbb{Z}^{\nu} .
$$

By using the Fourier transform, we can write the following representation of the Green function $G_{0}^{(\nu)}(\mathbf{x}-\mathbf{y} ; z)$ of the $\nu$-dimensional Laplacian $(2.2)$ for $d=\nu$ :

$$
G_{0}^{(\nu)}(\mathbf{x}-\mathbf{y} ; z)=\int_{\mathbb{T}^{\nu}} d \mathbf{k} \frac{e^{2 i \pi \mathbf{k} \cdot(\mathbf{x}-\mathbf{y})}}{E_{\nu}(\mathbf{k})-z}
$$

where

$$
E_{\nu}(\mathbf{k})=-\sum_{i=1}^{\nu} \cos k_{i}, \quad\left(k_{1}, \ldots, k_{\nu}\right)=\mathbf{k} \in \mathbb{T}^{\nu} .
$$

These formulas allow us to define the following operators on $l^{2}(S)$ :

$$
\gamma_{0}(z)=P_{S} G_{0}(z) P_{S}, \quad b(z)=\left(g \gamma_{0}(z)-i\right)\left(g \gamma_{0}(z)+i\right)^{-1},
$$

and to prove that:

$$
\|b(z)\|<1, \quad \Im z \neq 0,
$$


Lemma 2.1. The operator $t(z)$ defined by (2.5)-(2.8) and acting in $P_{S} l^{2}\left(\mathbb{Z}^{d}\right)=l^{2}(S)$ can be represented in the form:

$$
t(z)=g(1-\sigma u)(1-\sigma b(z) u)^{-1}\left(g \gamma_{0}(z)+i\right)^{-1},
$$

or

$$
t(z)=g\left(g \gamma_{0}(z)+i\right)^{-1}\left[1-2 i \sigma u \sum_{l=0}^{q-1}(\sigma b(z) u)^{l}\left(1-(\sigma b(z) u)^{q}\right)^{-1}\left(g \gamma_{0}(z)+i\right)^{-1}\right],
$$

where $\sigma=e^{-2 i \pi \omega}$ and $q \geq 1$ is an integer.

Proof. By using the Euler formula for the function $x \rightarrow \tan (x)$ and the notations introduced above, we obtain that:

$$
v=\frac{g}{i} \cdot \frac{1-\sigma u}{1+\sigma u}
$$

This observation and a simple algebra lead to (2.16) and (2.17).

Proposition 2.1. Let $H$ be the operator defined by (2.1), (2.2) and (1.1). Then its resolvent $G(z)=(H-z)^{-1}$ can be represented as follows for $\Im z>0$ :

$$
\begin{aligned}
G(z) & =G_{0}(z)-g G_{0}(z) P_{S}\left(g \gamma_{0}(z)+i\right)^{-1} P_{S} G_{0}(z)+2 i g G_{0}(z) P_{S}\left(g \gamma_{0}(z)+i\right)^{-1} \\
& \times \sigma u \sum_{l=0}^{q-1}(\sigma b(z) u)^{l}\left(1-(\sigma b(z) u)^{q}\right)^{-1}\left(g \gamma_{0}(z)+i\right)^{-1} P_{S} G_{0}(z)
\end{aligned}
$$

where $\sigma=e^{-2 i \pi \omega}, q \geq 1$ is an integer, and operators $G_{0}(z), \gamma_{0}(z), u$, and $b(z)$ are defined in (2.6), (2.9) and in (2.14).

Proof. The proposition follows easily from (2.5), Lemma 2.1 and from bound (2.15). Remark. Integrate formula (2.19) with respect to $\omega \in[0,1)$ and denote this operation by $\langle\cdots\rangle$. We obtain:

$$
\langle G(z)\rangle=G_{0}(z)-g G_{0}(z) P_{S}\left(g \gamma_{0}(z)+i\right)^{-1} P_{S} G_{0}(z) .
$$

In view of the general formula $t(z)=\left(v^{-1}+\gamma_{0}(z)\right)^{-1}$, valid for any surface potential $v$, we can interpret the equality $\langle t(z)\rangle=g\left(g \gamma_{0}(z)+i\right)^{-1}=\left(-(i g)^{-1}+\gamma_{0}(z)\right)^{-1}$ as the fact that $\langle G(z)\rangle$ is the resolvent of the Schrödinger operator whose surface potential is the complex constant $-i g$. Similar fact is known in the case of the volume potential (2.4), i.e. for the case $S=\mathbb{Z}^{d}[2,19]$.

We will obtain now a useful representation for the Green function $G(\mathbf{x}, \mathbf{y} ; z):=$ $(H-z)^{-1}(\mathbf{x}, \mathbf{y})$ of $H$.

Lemma 2.2. Let $b(z)$ and $u$ be the operators defined in (2.14) and (2.9). Then for any integer $m \geq 1$,

$$
\left(\left(b \widehat{(z) u)^{m}} \varphi\right)\left(k_{2}\right)=\left(\prod_{l=0}^{m-1} \hat{b}\left(k_{2}+l \alpha ; z\right)\right) \hat{\varphi}\left(k_{2}+m \alpha\right), \quad k_{2} \in \mathbb{T}^{d_{2}},\right.
$$

where for $\varphi \in l^{2}\left(\mathbb{Z}^{d_{2}}\right)$, $\hat{\varphi}$ denotes its Fourier transform,

$$
\hat{b}\left(k_{2} ; z\right)=\frac{\left(g \hat{\gamma}_{0}\left(k_{2} ; z\right)-i\right)}{\left(g \hat{\gamma}_{0}\left(k_{2} ; z\right)+i\right)}, \quad \hat{\gamma}_{0}\left(k_{2} ; z\right)=\int_{\mathbb{T}^{d_{1}}} \frac{d k_{1}}{E_{d}\left(\left(k_{1}, k_{2}\right)\right)-z} .
$$


Proof. We first note that the operator $\gamma_{0}(z)$ of $(2.14)$ acts on $l^{2}\left(\mathbb{Z}^{d_{2}}\right)$. Its matrix

$$
\gamma_{0}\left(x_{2}-y_{2} ; z\right)=G_{0}^{d}\left(\left(0, x_{2}\right)-\left(0, y_{2}\right) ; z\right)
$$

depends only on the difference $\left(x_{2}-y_{2}\right)$ and in view of (2.12) is,

$$
\gamma_{0}\left(x_{2} ; z\right)=\int_{\mathbb{T}^{d_{2}}} d k_{2} e^{2 i \pi k_{2} \cdot x_{2}} \int_{\mathbb{T}^{d_{1}}} \frac{d k_{1}}{E_{d}(\mathbf{k})-z}
$$

or

$$
\gamma_{0}\left(x_{2} ; z\right)=\int_{\mathbb{T}^{d_{2}}} d k_{2} e^{2 i \pi k_{2} \cdot x_{2}} \hat{\gamma}_{0}\left(k_{2} ; z\right)
$$

where $\hat{\gamma}_{0}\left(k_{2} ; z\right)$ is given in (2.21). This and (2.14) imply that the operator $b(z)$ also has the matrix depending on the difference $\left(x_{2}-y_{2}\right)$, thus it is the multiplication operator by $\hat{b}\left(k_{2} ; z\right)$ of $(2.21)$ after the Fourier transformation. Besides, it follows from (2.9 that after the Fourier transformation the operator $u$ is the shift by $\alpha$ i.e.

$$
\widehat{u \varphi}\left(k_{2}\right)=\hat{\varphi}\left(k_{2}+\alpha\right)
$$

The above facts imply

Theorem 2.1. Let $H$ be the operator defined by (2.1), (2.2) and (1.1). Then the Green function $G(\mathbf{x}, \mathbf{y} ; z)=(H-z)^{-1}(\mathbf{x}, \mathbf{y})$ is given by the formula:

$$
\begin{aligned}
G(\mathbf{x}, \mathbf{y} ; z)= & G_{0}^{(d)}(\mathbf{x}-\mathbf{y} ; z)-\sum_{m=0}^{\infty} \int_{\mathbb{T}^{d_{2}}} d k_{2} e^{2 i \pi k_{2} \cdot\left(x_{2}-y_{2}\right)} t_{m}\left(k_{2} ; z\right) \\
& \times G_{0}^{\left(d_{1}\right)}\left(x_{1} ; z-E_{d_{2}}\left(k_{2}\right)\right) G_{0}^{\left(d_{1}\right)}\left(y_{1} ; z-E_{d_{2}}\left(k_{2}+m \alpha\right)\right) e^{-2 i \pi m \alpha \cdot y_{2}},
\end{aligned}
$$

where $t_{m}\left(k_{2} ; z\right)$ is defined by

$$
-\frac{g}{g \hat{\gamma}_{0}\left(k_{2} ; z\right)+i} \begin{cases}-1, & m=0 \\ 2 i \sigma\left(g \hat{\gamma}_{0}\left(k_{2}+\alpha ; z\right)+i\right)^{-1}, & m=1 \\ 2 i \sigma^{m}\left(g \hat{\gamma}_{0}\left(k_{2}+m \alpha ; z\right)+i\right)^{-1} \prod_{l=0}^{m-1} \hat{b}\left(k_{2}+l \alpha ; z\right), & m \geq 2\end{cases}
$$

$G_{0}^{\left(d_{1}\right)}\left(x_{1} ; z\right)$ is the Green function (2.12) of the $d_{1}$-dimensional Laplacian, $E_{d_{2}}\left(k_{2}\right)$ is defined in (2.13) for $\nu=d_{2}$, and $\hat{\gamma}_{0}\left(k_{2} ; z\right)$ and $\hat{b}\left(k_{2} ; z\right)$ are defined in (2.14) and in (2.21).

Besides, the (generalized) kernel of the operator $T$ of (2.5) and of Lemma 2.1 in the momentum representation has the form:

$$
T(\mathbf{k}, \mathbf{p} ; z)=\sum_{m=0}^{\infty} t_{m}\left(k_{2} ; z\right) \delta\left(k_{2}+m \alpha-p_{2}\right),
$$

where $t_{m}\left(k_{2} ; z\right)$ is defined in (2.26). In particular, the kernel is independent of the components $k_{1}, p_{1} \in \mathbb{T}^{d_{1}}$ of its arguments $\mathbf{k}, \mathbf{p} \in \mathbb{T}^{d}$. 
Proof. According to (2.15), $\|b(z)\|<1$ if $\Im z \neq 0$. Hence we can write the operator $(1-\sigma b u)^{-1}$ in $(2.16)$ for $q=1$ as the Neumann-Liouville series in power of $\sigma b u$. Applying to the each term of the series inequality (2.15), we get (2.25) after a simple algebra. Formula (2.27) follows from (2.5) and (2.25).

Remark. Formulas (2.25) and (2.27) have to be compared with the formulas for respective quantities in the cases of the point potential $V(\mathbf{x})=v \delta(\mathbf{x}),\left(d_{2}=0\right)$ and of the constant surface potential $V(\mathbf{x})=v \delta\left(x_{1}\right), v=$ const. In the first case we have:

$$
G(\mathbf{x}, \mathbf{y} ; z)=G_{0}^{(d)}(\mathbf{x}-\mathbf{y} ; z)-\frac{v}{1+v G_{0}^{(d)}(0 ; z)} G_{0}^{(d)}(\mathbf{x} ; z) G_{0}^{(d)}(\mathbf{y} ; z)
$$

and

$$
T(\mathbf{k}, \mathbf{p} ; z)=\frac{v}{1+v G_{0}^{(d)}(0 ; z)}
$$

while in the second case:

$$
\begin{aligned}
G(\mathbf{x}, \mathbf{y} ; z)=G_{0}^{(d)}(\mathbf{x}-\mathbf{y} ; z)- & \int_{\mathbb{T}^{d_{2}}} d k_{2} \frac{v e^{2 i \pi k_{2} \cdot\left(x_{2}-y_{2}\right)}}{1+v G_{0}^{\left(d_{1}\right)}\left(0 ; z-E_{d_{2}}\left(k_{2}\right)\right)} \\
& \times G_{0}^{\left(d_{1}\right)}\left(x_{1} ; z-E_{d_{2}}\left(k_{2}\right)\right) G_{0}^{\left(d_{1}\right)}\left(y_{1} ; z-E_{d_{2}}\left(k_{2}\right)\right),
\end{aligned}
$$

and

$$
T(\mathbf{k}, \mathbf{p} ; z)=\frac{v \delta\left(k_{2}-p_{2}\right)}{1+v G_{0}^{\left(d_{1}\right)}\left(0 ; z-E_{d_{2}}\left(k_{2}\right)\right)},
$$

Formulas (2.25) and (2.27) are the basic tools of spectral and scattering analysis of the operator (2.1) presented in this paper. An advantage of the formulas is that they are valid for all values of the spectral parameter $z=E+i \varepsilon$, up to the real values $z=E \pm i 0,|E|<d$ in the case of irrational $\alpha$ 's (quasi-periodic in $x_{2}$ potential (1.1)), and for all $E \in \mathbb{R}$ in the case of rational $\alpha$ 's (periodic in $x_{2}$ potential (1.1)). We discuss these cases in Sections 2 and 3 respectively.

\section{Quasi Periodic Case}

In this section we assume that the vector $\alpha \in \mathbb{R}^{d_{2}}$ from (1.1) has rationally independent components, i.e. the relation $\alpha_{1} r_{1}+\cdots+\alpha_{d_{2}} r_{d_{2}}=0$ with rational coefficients $r_{1}, \ldots, r_{d_{2}}$ implies that all these coefficients are zero.

Besides, we restrict ourselves to the interval $[-d, d]$ of the spectral axis. We first prove

Theorem 3.1. Let $H=H_{0}+V$ be the operator defined by (2.1), (2.2) and (1.1). Then its spectrum $\sigma(H)$ contains the interval $[-d, d]=\sigma\left(H_{0}\right)$ for all $g \in \mathbb{R}, \alpha \in \mathbb{R}^{d_{2}}$ and $\omega \in[0,1]$.

The proof is a direct application of the H. Weyl criterion.

Theorem 3.2. Let $H=H_{0}+V$ be the self-adjoint operator defined by (2.2) and (1.1) in which $\alpha \in \mathbb{R}^{d_{2}}$ has rationally independent components. Then the spectrum of $H$ on the interval $[-d, d]$ is purely absolutely continuous. 
The proof of the theorem is based on Theorem 2.1, and on the relation

$$
\lim _{m \rightarrow \infty}\left|\prod_{l=0}^{m-1} \hat{b}\left(k_{2}+l \alpha ; E+i 0\right)\right|=\exp \left\{\int_{\mathbb{T}^{d_{2}}} d q_{2} \log \left|\hat{b}\left(q_{2} ; E+i 0\right)\right|\right\},
$$

valid uniformly in $k_{2} \in \mathbb{T}^{d_{2}}$ (see e.g [1]). It can be shown that if $|E| \leq d-\gamma, \gamma>0$, then the integral in the r.h.s. is negative, and the product in the l.h.s. is exponentially decaying in $m$ as $m \rightarrow \infty$, implying the convergence of the series in (2.5). This reveals a fairly simple mathematical mechanism responsible for the absolutely continuous spectrum for the "subspace" potential (1.1) with $d_{1} \geq 1$ (recall that in the "volume" case $d_{1}=0, d_{2}=d$, the absolutely continuous spectrum is absent, moreover if $\alpha$ is Diophantine then the spectrum is pure point $[2,15])$. The mechanism is the positiveness of the imaginary part of $\hat{\gamma}_{0}\left(k_{2} ; E+i 0\right)=G_{0}^{\left(d_{1}\right)}\left(0, E+i 0-E\left(k_{2}\right)\right)$ in certain domain of $\left(E, k_{2}\right)$. This is most transparent in the "genuine surface" case $d_{1}=1$, where $G_{0}^{(1)}(0, E+i 0)$ is pure imaginary if $|E|<1$ and is pure real if $|E| \geq 1$, (see formula (3.13) below). In the latter case $\left.\mid \hat{b}\left(q_{2} ; E+i 0\right)\right) \mid=1$ and the series (2.25) diverges for a dense set of energies (see [2]). This leads to the pure point spectrum everywhere outside of the spectrum $\sigma\left(H_{0}\right)$ of the Laplacian (similarly to the volume case [2], where the analogue of $\hat{\gamma}_{0}\left(k_{2} ; E\right)$ in $(2.21)$ is real for all $E \in \mathbb{R}$ and the whole spectrum is pure point). In the former case $\left.\mid \hat{b}\left(k_{2} ; E+i 0\right)\right) \mid$ is strictly less than 1 for any $E \in(-d, d)$ on an open set of $k_{2} \in \mathbb{T}^{d_{2}}$, the series is convergent and the spectrum inside of $\sigma\left(H_{0}\right)$ is pure absolutely continuous.

As usual in spectral theory involving the absolutely continuous spectrum and in scattering theory, a fact of primary interest is existence and completeness of wave operators $\Omega_{\mp}=s-\lim _{t \rightarrow \mp \infty} e^{i t H_{0}} e^{-i t H} \mathcal{E}_{0}(\Delta)$, where $\mathcal{E}_{0}$ is the resolution of identity of $H_{0}$, and $\Delta$ is an interval of the spectral axis. In the next theorem we prove existence and completeness of wave operators in our case.

We mention that in papers $[4,5,6,9]$, the scattering theory was developed for the operator $H_{1}$, acting in $l^{2}\left(\mathbb{Z}_{+}^{d}\right)$ (with $\mathbb{Z}_{+}^{d}=\left\{\left(x_{1}, x_{2}\right) \in \mathbb{Z}^{d} ; x_{1} \geq 0, \mathbf{x}_{2} \in \mathbb{Z}^{d-1}\right\}$ ), and defined as:

$$
\left(H_{1} \Psi\right)(\mathbf{x})= \begin{cases}-1 / 2(\Delta \Psi)(\mathbf{x}), & x_{1} \geq 1 \\ -1 / 2 \Psi\left(1, x_{2}\right)-1 / 2 \sum_{\left|x_{2}-y_{2}\right|=1} \Psi\left(0, y_{2}\right)+v\left(x_{2}\right) \Psi\left(0, x_{2}\right), & x_{1}=0\end{cases}
$$

for certain random and almost periodic $v^{\prime}$ s. The operator can be viewed as defined by the boundary value problem for the discrete Laplacian in $l^{2}\left(\mathbb{Z}_{+}^{d}\right)$ with the boundary condition $\Psi\left(-1, x_{2}\right)=v\left(x_{2}\right) \Psi\left(0, x_{2}\right), x_{2} \in \mathbb{Z}^{d-1}$. The "unperturbed" operator $H_{0}$ here is the discrete Dirichlet Laplacian, corresponding to $v \equiv 0$ in (3.2). The operator $H_{1}$ is closely related to our operator $H$ of (2.1) for the surface case $d_{1}=1$, $d_{2}=d-1$.

Theorem 3.3. Under the conditions of the Theorem 3.2, the wave operators $\Omega_{ \pm}$for the pair $\left(H, H_{0}\right)$, defined by (2.1), (2.2), and (1.1), exist and are complete for any closed interval $\Delta=[a, b] \subset(-d, d)$.

The proof combines a general argument of [9], and Theorem 2.1. 
The next theorem presents an explicit form of the generalized eigenfunctions of the operator $H$ on the interval $(-d, d)$ in the case of rationally independent frequencies $\alpha_{1}, \ldots, \alpha_{d_{2}}$ in (2.3). The proof of the theorem is based on formulas (2.25), and (2.26) for the Green function of the operator $H$, and on relation (3.1).

Theorem 3.4. Let $G(\mathbf{x}, \mathbf{y}: z)$ be the Green function of the operator $H$ defined by (2.1), (2.2), and (1.1). Set

$$
\begin{gathered}
G(\mathbf{x}, \mathbf{k} ; z)=\sum_{\mathbf{y} \in z^{d}} G(\mathbf{x}, \mathbf{y} ; z) e^{2 i \pi \mathbf{k} \cdot \mathbf{y}}, \quad \mathbf{k} \in \mathbb{T}^{d} \\
\Psi_{z}(\mathbf{x}, \mathbf{k})=\left(E_{d}(\mathbf{k})-z\right) G(\mathbf{x}, \mathbf{k} ; z)
\end{gathered}
$$

and

$$
\dot{\mathbb{T}}^{d_{2}}=\mathbb{T}^{d_{2}} \backslash\{\underbrace{(0,0, \ldots, 0)}, \underbrace{d_{2}-\text { times }}\left(\begin{array}{c}
d_{2}-\text { times } \\
(\pi, \ldots, \pi)
\end{array}\right\} ; \quad \dot{\mathbb{T}}^{d}=\dot{\mathbb{T}}^{d_{2}} \times \mathbb{T}^{d_{1}} .
$$

Then, for $z=E_{d}(\mathbf{k}) \mp i \varepsilon$, the limits:

$$
\Psi_{ \pm}(\mathbf{x}, \mathbf{k}):=\left.\lim _{\varepsilon \rightarrow \pm 0} \Psi_{z}(\mathbf{x}, \mathbf{k})\right|_{z=E_{d}(\mathbf{k}) \mp i \varepsilon}=\lim _{\varepsilon \rightarrow \pm 0}( \pm i) \varepsilon G\left(\mathbf{x}, \mathbf{k} ;\left(E_{d}(\mathbf{k}) \mp i \varepsilon\right)\right)
$$

exist for all $\mathbf{k} \in \dot{\mathbb{T}}^{d}$, are bounded in $\mathbf{x} \in \mathbb{Z}^{d}$ for all $\mathbf{k} \in \dot{T}^{d}$ and are continuous in $\mathbf{k}$ varying in any compact set of $\dot{\mathbb{T}}^{d}$. They are given by the formula

$$
\begin{aligned}
\Psi_{ \pm}(\mathbf{x}, \mathbf{k})=e^{2 i \pi \mathbf{k} \cdot \mathbf{x}}+ & \sum_{m=0}^{\infty} t_{m}\left(k_{2}-m \alpha ; z\right) \\
& \times G_{0}^{\left(d_{1}\right)}\left(x_{1} ; E_{d}(\mathbf{k}) \mp i 0-E_{d_{2}}\left(k_{2}-m \alpha\right) e^{2 i \pi\left(k_{2}-m \alpha\right) \cdot x_{2}} .\right.
\end{aligned}
$$

Moreover, we have:

(i) $\Psi_{ \pm}(\mathbf{x}, \mathbf{k})$ satisfy the Schrödinger equation:

$$
\left(\left(H_{0}+V\right) \Psi_{ \pm}\right)(\mathbf{x}, \mathbf{k})=E_{d}(\mathbf{k}) \Psi_{ \pm}(\mathbf{x}, \mathbf{k}) .
$$

(ii) $\Psi_{ \pm}$are the unique solutions of the integral equations:

$$
\Psi_{ \pm}(\mathbf{x}, \mathbf{k})=e^{2 i \pi \mathbf{k} \cdot \mathbf{x}}-\sum_{\mathbf{y} \in \mathbb{Z}^{d}} G_{0}^{(d)}\left(\mathbf{x}-\mathbf{y} ; E_{d}(\mathbf{k}) \mp 0\right) V(\mathbf{y}) \Psi_{ \pm}(\mathbf{y}, \mathbf{k}) .
$$

in the class of sequences $\{\Psi(\mathbf{x})\}_{\mathbf{x} \in \mathbb{Z}^{d}}$ whose restrictions $\left\{\psi_{2}\left(x_{2}\right):=\Psi\left(0, x_{2}\right)\right\}_{x_{2} \in \mathbb{Z}^{d_{2}}}$ and $\left\{\left(1+\sigma e^{-2 i \pi \alpha \cdot x_{2}}\right) \psi_{2}\left(x_{2}\right)\right\}_{x_{2} \in \mathbb{Z}^{d_{2}}}$ are representable as the Fourier transform of measures of bounded variation on $\mathbb{T}^{d_{2}}$, and the sum of the l.h.s. of (3.8) are understood as the Fourier transform of the product of the respective function and the measure.

(iii) The families $\left\{\Psi_{ \pm}(., \mathbf{k})\right\}_{\mathbf{k} \in \dot{T}^{d}}$ are orthonormalized, i.e. if for any continuous function $\hat{\Phi}(\mathbf{k})$ having compact support in $\dot{\mathbb{T}}^{d}$ we set

$$
\Phi_{ \pm}(\mathbf{x})=\int_{\dot{T}^{d}} \Psi_{ \pm}(\mathbf{x}, \mathbf{k}) \hat{\Phi}(\mathbf{k}) d \mathbf{k},
$$

then for any two such functions $\hat{\Phi}^{(1)}$ and $\hat{\Phi}^{(2)}$ we have:

$$
\sum_{\mathbf{x} \in \mathbb{Z}^{d}} \Phi_{ \pm}^{1}(\mathbf{x}) \overline{\Phi_{ \pm}^{2}(\mathbf{x})}=\int_{\dot{\mathbb{T}}^{d}} d \mathbf{k} \hat{\Phi}^{(1)}(\mathbf{k}) \overline{\hat{\Phi}^{(2)}(\mathbf{k})}
$$


(iv) The functions $\Psi_{ \pm}: \mathbb{Z}^{d} \times \dot{\mathbb{T}}^{d} \rightarrow \mathbb{C}$ are the kernels of the wave operators $\Omega_{ \pm}$, whose existence and completeness are proved in Theorem 3.3, i.e. for any $\Phi \in l^{2}\left(\mathbb{Z}^{d}\right)$ :

$$
\left(\Omega_{ \pm} \Phi\right)(\mathbf{x})=\int_{\dot{\mathbb{T}}^{d}} \Psi_{ \pm}(\mathbf{x}, \mathbf{k}) \hat{\Phi}(\mathbf{k}) d \mathbf{k}
$$

where $\hat{\Phi}$ is the Fourier transform of $\Phi$.

Remarks. 1) $\Psi_{ \pm}(\mathbf{x}, \mathbf{k})$ are analogous of the Sommerfeld solutions, known in the scattering theory (i.e. in the case of a potential decaying in all directions) and providing a complete set of generalized eigenfunctions in the part of the spectrum that coincides with the spectrum of the Laplacian [16, 18]. Likewise, (3.8) is an analogue of the Lippmann-Schwinger equation of scattering theory.

2) According to formula (3.6), $\Psi_{ \pm}(\mathbf{x}, \mathbf{k})$ depends on the component $x_{2} \in \mathbb{Z}^{d_{2}}$ of $\mathbf{x}=\left(x_{1}, x_{2}\right)$ via the product of $e^{i k_{2} \cdot x_{2}}$ and of a 1-periodic function of the argument $\alpha x_{2}$, i.e. of a quasi periodic function of $x_{2} \in \mathbb{Z}^{d_{2}}$. This fact is in agreement with the widely accepted in the spectral theory expectation, according to which generalized eigenfunctions of absolutely continuous spectrum of differential and finite difference operators with almost periodic coefficients have the "almost Floquet-Bloch" form of the product of the plane wave and of an almost periodic function with the same set of the Fourier frequencies as the coefficients.

3 ) According to (2.12)) the Green function $G_{0}^{(\nu)}(\mathbf{x} ; E+i 0)$ of the $\nu$-dimensional Laplacian decays exponentially if $|E|>\nu$ and decays as $\left|x_{1}\right|^{\frac{\nu-1}{2}}$ if $|E|<\nu$ and $\nu \geq 2$. In the one dimensional case $G_{0}^{(1)}(x ; E+i 0)$ behaves as $e^{i \eta(E)|x|}$ for $|E|<1$, where $\eta(E)$ is a real valued function (see formula (3.13), and (3.14) below ). Since the expression $E_{d}(\mathbf{k})-E_{d_{2}}\left(k_{2}-m \alpha\right)$ assumes values inside of $\left(-d_{1}, d_{1}\right)$ as well as outside of this interval as $m$ varies, the Green function

$$
G_{0}^{\left(d_{1}\right)}\left(x_{1} ; E_{d}(\mathbf{k}) \mp i 0-E_{d_{2}}\left(k_{2}-m \alpha\right)\right),
$$

entering the expression (3.6), may be exponentially decaying or slowly decaying as $\left|x_{1}\right|^{\frac{\nu-1}{2}}$. Hence, despite that in the quasi periodic case the surface state are absent (see [10,9] for definitions of these generalized eigenfunctions), the "volume" generalized functions (3.6) contain both slowly decaying or even only oscillating in $\left|x_{1}\right|$ terms and exponentially decaying in $\left|x_{1}\right|$ terms. These terms can be interpreted as the waves reflected from the surfaces and propagating correspondingly inside the bulk and along the subspace $\mathbb{Z}^{d_{2}}$, the support of the quasi-periodic perturbation (strongly corrugated quasi-periodic surface in the case $d_{1}=1$ ). In other words, we can write:

$$
\Psi(\mathbf{x}, \mathbf{k})=e^{2 i \pi \mathbf{k} \cdot \mathbf{x}}+\Psi_{\text {vol }}(\mathbf{x}, \mathbf{k})+\Psi_{\text {surf }}(\mathbf{x}, \mathbf{k}) .
$$

The scattering interpretation (3.12) of generalized eigenfunctions (3.6) allows us to introduce transmission and reflection amplitudes. Consider the simplest case of $d_{1}=1$ and recall that:

$$
G_{0}^{(1)}\left(x_{1} ; z\right)=\frac{i e^{i \eta(z)\left|x_{1}\right|}}{\sin \eta(z)}
$$

where $-\cos \eta=z$, or

$$
\eta(z)=-i \log \left(-z+\sqrt{z^{2}-1}\right),
$$


and we use the branch of the logarithm that has the cut along the negative semi-axis and the branch of $\sqrt{z^{2}-1}$ fixed by the condition $\sqrt{z^{2}-1}=z\left(1+O\left(z^{-1}\right)\right), z \rightarrow \infty$. In particular $\Im \eta(z) \geq 0$ for $\Im z \geq 0$ and

$$
\eta(E+i 0) \in \begin{cases}(0, \pi), & |E|<1 \\ -i R_{+}, & E>1 \\ +i R_{-}, & E<-1\end{cases}
$$

Combining these formulas and (3.6), we can present $\Psi_{v o l}(\mathbf{x}, \mathbf{k})$ for $d_{1}=1$ as

$$
\Psi_{v o l}(\mathbf{x}, \mathbf{k})=\sum_{m} \Psi_{m}(\mathbf{k}) e^{i \eta_{m}(\mathbf{k})\left|x_{1}\right|+2 i \pi\left(k_{2}+\alpha m\right) x_{2}},
$$

where $\sum_{m}$ denotes the sum of those terms in (3.6) in which $\eta_{m}(\mathbf{k}):=\eta\left(q_{m}(\mathbf{k})\right)$ is real, and $q_{m}(\mathbf{k})=E_{d}(\mathbf{k})-E_{d-1}\left(k_{2}-m \alpha\right)$. Recall that the Sommerfeld solution in the one-dimensional scattering problem for the potential $v \delta\left(x_{1}\right), x_{1} \in \mathbb{Z}$, is

$$
\Psi\left(x_{1}, k_{1}\right)=e^{2 i \pi k_{1} x_{1}}-\frac{i v}{i v+\left|\sin \left(2 \pi k_{1}\right)\right|} e^{2 i \pi\left|k_{1}\right|\left|x_{1}\right|},
$$

and hence the quantities

$$
t\left(k_{1}\right)=\frac{\left|\sin \left(2 \pi k_{1}\right)\right|}{i v+\left|\sin \left(2 \pi k_{1}\right)\right|}, \quad r\left(k_{1}\right)=\frac{i}{i v+\left|\sin \left(2 \pi k_{1}\right)\right|}
$$

are the transmission and the reflection amplitudes in this problem. This makes natural to view

$$
t_{0}=1-\Psi_{0}, \quad r_{0}=\Psi_{0}
$$

as the transmission and the reflection amplitudes of the scattered by the surface potential (1.1) plane waves, propagating in direction $\left(k_{1}, k_{2}\right)$ of the incident wave and in the opposite direction, and to view $\Psi_{m}, m \geq 1$ as transmission and reflection coefficients of the scattered plane waves propagating in directions $\left(\eta_{m}, k_{2}-m \alpha\right)$ and $\left(-\eta_{m}, k_{2}-m \alpha\right)$ respectively.

This interpretation of the solutions (3.6) is in agreement with the form of the scattering matrix $\mathcal{S}$ in our case. We use the general formula ([16], formula (4.2.30):

$$
\mathcal{S}=1-\mathcal{T}, \quad \mathcal{T}=-2 i \pi \times s \cdot \lim _{\substack{\varepsilon_{1} \rightarrow 0 \\ \varepsilon_{2} \rightarrow 0}} \int_{\mathbb{R}} \delta_{\varepsilon_{2}}\left(H_{0}-\lambda\right) T\left(\lambda+i \varepsilon_{1}\right) \mathcal{E}_{0}(d E),
$$

where $\delta_{\varepsilon}(A)=(2 i \pi)^{-1}\left[(A+i \varepsilon)^{-1}-(A-i \varepsilon)^{-1}\right], T(z)$ is defined in $(2.5), \mathcal{E}_{0}$ is the resolution of identity of $H_{0}$, and the limits have to be carried out in the following order: first $\varepsilon_{1} \rightarrow 0$, second $\varepsilon_{2} \rightarrow 0$. Formula (3.16) implies that for any sufficient smooth function $\hat{\Phi}(\mathbf{k})$ :

$$
(\mathcal{T} \hat{\Phi})(\mathbf{k})=-2 i \pi \times s \cdot \lim _{\substack{\varepsilon_{1} \rightarrow 0 \\ \varepsilon_{2} \rightarrow 0}} \int_{T^{d}} d \mathbf{p} \delta_{\varepsilon_{2}}\left(E_{d}(\mathbf{k})-E_{d}(\mathbf{p})\right) T\left(\mathbf{k}, \mathbf{p} ; E_{d}(\mathbf{p})+i \varepsilon_{1}\right) \hat{\Phi}(\mathbf{p}) .
$$


This formula and formula (2.27) for the kernel of the $T$-operator imply that the generalized kernel $\mathcal{T}(\mathbf{k}, \mathbf{p})$ of the $\mathcal{T}$-matrix of (3.16) is:

$$
\begin{aligned}
\mathcal{T}(\mathbf{k}, \mathbf{p}) & =-2 i \pi \delta\left(E_{d}(\mathbf{k})-E_{d}(\mathbf{p})\right) T\left(\mathbf{k}, \mathbf{p} ; E_{d}(\mathbf{p})+i 0\right) \\
& =-2 i \pi \delta\left(E_{d}(\mathbf{k})-E_{d}(\mathbf{p})\right) \sum_{m=0}^{\infty} t_{m}\left(k_{2} ; E_{d}(\mathbf{k})+i 0\right) \delta\left(k_{2}+m \alpha-p_{2}(3.17)\right.
\end{aligned}
$$

The next theorem shows that the family of the "Sommerfeld-like" solutions constructed in the previous theorem is complete on the interval $(-d, d)$, the spectrum of the discrete Laplacian (2.2). The proof of the theorem combines again a version of standard means of the scattering theory, the resolvent equation in particular (see e.g. $[16,18])$, and the representation $(2.25),(2.26)$ of the Green function of the operator $H$.

Theorem 3.5. Let $H=H_{0}+V$ be the self-adjoint operator on $l^{2}\left(\mathbb{Z}^{d}\right)$, defined by (2.2) and (1.1). Then the family $\left\{\Psi_{z}(\mathbf{x}, \mathbf{k}) ; \mathbf{x} \in \mathbb{Z}^{d}\right\}_{\mathbf{k} \in \dot{\mathbb{T}}^{d}}$ defined in Theorem 3.4 (see (3.3), (3.5), (3.6) is the complete system of generalized eigenfunctions of $H$ in the part $(-d, d)$ of the spectrum i.e.:

(i) for any $\Phi \in l^{2}\left(\mathbb{Z}^{d}\right)$ the series:

$$
\hat{\Phi}(\mathbf{k})=\sum_{\mathbf{x} \in \mathbb{Z}^{d}} \Psi_{ \pm}(\mathbf{x}, \mathbf{k}) \Phi(\mathbf{x})
$$

converges in $l^{2}\left(\mathbb{Z}^{d}\right)$;

(ii) if $\mathcal{E}(\Delta)$ is the spectral projection of $H$, corresponding to the closed interval $\Delta=[a, b] \subset(-d, d)$, then

$$
\|\mathcal{E}(\Delta) \Phi\|^{2}=\int_{\left\{\mathbf{k} \in \mathbb{T}^{d}: E(\mathbf{k}) \in \Delta\right\}}\left|\hat{\Phi}_{ \pm}(\mathbf{k})\right|^{2} d \mathbf{k}
$$

where $E(\mathbf{k})$ is defined in (2.13));

(iii) the following relation is valid:

$$
\|H \mathcal{E}(\Delta) \Phi\|^{2}=\int_{\left\{\mathbf{k} \in \mathbb{T}^{d}: E(\mathbf{k}) \in \Delta\right\}}\left|E_{d}(\mathbf{k}) \hat{\Phi}_{ \pm}(\mathbf{k})\right|^{2} d \mathbf{k} .
$$

\section{Periodic Case}

In this section we discuss briefly the case where the vector $\alpha$ in (1.1) has commensurate components, i.e. the potential (1.1) is periodic in $x_{2}$. For the sake of technical multiplicity we consider here the two dimensional case $d_{1}=d_{2}=1$, i .e. the case of the "line" periodic potential in which

$$
\alpha=p / q,
$$


where $p$ and $q$ are mutually simple integers and $q \geq 2$. Our analysis is based on the formula (2.19) for the resolvent of $H$ of (2.1), whose coordinate version is ( $\mathrm{cf}(2.25)-$ $(2.26))$ :

$$
\begin{aligned}
G(\mathbf{x}, \mathbf{y} ; z)=G_{0}^{(1)} & (\mathbf{x}-\mathbf{y} ; z)-\sum_{m=0}^{q-1} \int_{T^{1}} d k_{2} e^{2 i \pi k_{2} \cdot\left(x_{2}-y_{2}\right)} t_{m}\left(k_{2} ; z\right) \\
& \times G_{0}^{(1)}\left(x_{1} ; z+\cos k_{2}\right) G_{0}^{(1)}\left(y_{1} ; z+\cos \left(k_{2}+m \alpha\right)\right) e^{-2 i \pi m \alpha \cdot y_{2}}
\end{aligned}
$$

where $t_{m}\left(k_{2} ; z\right)$ is equal to

$$
-\frac{g}{g \hat{\gamma}_{0}\left(k_{2} ; z\right)+i} \begin{cases}-1, & m=0 \\ \frac{1}{1-P_{q}\left(k_{2} ; z\right)} \cdot \frac{2 i \sigma}{g \hat{\gamma}_{0}\left(k_{2}+\alpha ; z\right)+i}, & m=1, \\ \frac{1}{1-P_{q}\left(k_{2} ; z\right)} \cdot \frac{2 i \sigma}{g \hat{\gamma}_{0}\left(k_{2}+m \alpha ; z\right)+i} P_{m-1}\left(k_{2}+l \alpha ; z\right), & m \geq 2,\end{cases}
$$

$G_{0}^{(1)}\left(x_{1} ; z\right)$ is the Green function (2.12) of the 1-dimensional Laplacian, $\hat{\gamma}_{0}\left(k_{2} ; z\right)$, and $\hat{b}\left(k_{2} ; z\right)$ are defined in $(2.14)$ and in $(2.21)$, and

$$
P_{m}\left(k_{2} ; z\right)=\sigma^{m} \prod_{l=0}^{m-1} \hat{b}\left(k_{2}+l \alpha ; z\right), \quad \forall k_{2} \in T^{1}, m=1, \ldots, q .
$$

By using this formula, we show first that the spectrum of $H$ is the set

$$
\sigma(H)=[-2,2] \cup\left\{E \in \mathbb{R}: \exists k_{2} \in \mathbb{T}^{1}, P_{q}\left(k_{2}, E\right)=1\right\} .
$$

If $q$ is big enough, then the equation $P_{q}\left(k_{2}, E\right)=1$ has $q$ solutions: $E_{1}\left(k_{2}\right)<$ $E_{1}\left(k_{2}\right)<\cdots<E_{q}\left(k_{2}\right), k_{2} \in \mathbb{T}_{1}$, thus the second term in (4.5) consists of $q$ components:

$$
\left\{E \in \mathbb{R}: \exists k_{2} \in \mathbb{T}^{1}, P_{q}\left(k_{2}, E\right)=1\right\}=\cup_{l=1}^{q} \Delta_{l},
$$

where

$$
\Delta_{l}=\left\{E: \exists k_{2} \in \mathbb{T}^{1}, E_{l}\left(k_{2}\right)=E\right\} .
$$

All the intervals $\Delta_{l}$ except possible the first one lie outside of the interval $[-2,2]$, their width is exponentially small in $q$ and the distance between the neighbor $\Delta$ 's is of the order $1 / q$. Moreover, the intervals $\Delta_{l}$ tend in a natural sense to eigenvalues of the operator $H$ with an irrational Diophantine $\alpha$ as $p \rightarrow \infty, q \rightarrow \infty, p / q \rightarrow \alpha$ (recall that, according to [13], for such $\alpha$ 's the spectrum of $H$ is pure point and dense).

Next we show that the whole spectrum of $H$ is purely absolute continuous. As for the generalized eigenfunctions, they can be divided in two families with respect to their dependence on $x_{1}$. The first family consists of solutions of the Schrödinger equation, similar to the solutions (3.6). They are of the Floquet-Bloch form in $x_{2}$ with respective quasimomentum varying in the interval $(0,1 / q]$ and are the plane waves in $x_{1}$. These eigenfunctions correspond to the values of the spectral parameter belonging to $[-d, d]$, i.e. to the first term in (4.5). We will call them the volume states and will denote them $\Psi_{ \pm}^{(v)}(\mathbf{x}, \mathbf{k})$. The second family has no analogue in the 
quasi periodic case of the previous section. The family consists of the solutions that are of the Floquet-Bloch form in $x_{2}$ but decay exponentially in $x_{1}$ as $x_{1} \rightarrow \infty$. They correspond to the values of the spectral parameter belonging to the set (4.6), (4.7), i.e. to the second term in (4.5). We will call them the surface states and will denote them $\Psi_{ \pm}^{(s)}\left(\mathbf{x}, k_{2}\right)$. Likewise, the intervals $\Delta_{l}, l=1, \ldots, q$ will be called the surface bands.

The generalized eigenfunctions of both families are orthonormalized in the sense of relation (3.10), and the union of the two families is complete, i.e. if for any $\Phi \in l_{2}\left(\mathbb{Z}^{2}\right)$ we set

$$
\hat{\Phi}_{ \pm}^{(v)}(\mathbf{k})=\sum_{\mathbf{x} \in \mathbb{Z}^{2}} \Psi_{ \pm}^{(v)}(\mathbf{x}, \mathbf{k}) \Phi(\mathbf{x})
$$

and

$$
\hat{\Phi}_{ \pm}^{(s)}\left(k_{2}\right)=\sum_{\mathbf{x} \in \mathbb{Z}^{2}} \Psi_{ \pm}^{(s)}\left(\mathbf{x}, k_{2}\right) \Phi(\mathbf{x})
$$

and if $\mathcal{E}$ is, as above, the resolution of identity of the operator $H$, then

$$
\begin{aligned}
\|\mathcal{E}(\Delta) \Phi\|^{2} & =\int_{\left\{\mathbf{k} \in \mathbb{T}^{2}: E(\mathbf{k}) \in \Delta\right\}} d \mathbf{k}\left|\hat{\Phi}_{ \pm}^{(v)}(\mathbf{k})\right|^{2} \\
& +\sum_{l}^{q-1} \int_{\left\{k_{2} \in \mathbb{T}^{1}: E_{l}\left(k_{2}\right) \in \Delta_{l}\right\}} d k_{2}\left|\hat{\Phi}_{ \pm}^{(s)}\left(k_{2}\right)\right|^{2},
\end{aligned}
$$

As was mentioned above, there exists at most one band of surface states that intersects the interval $[-2,2]$, i.e. the spectrum of the two dimensional discrete Laplacian. This coexistence of the volume states and the surface states on the interval $[-2,2]$ is possible under rather special conditions on the parameters $g$ and $\omega$ in the potential (1.1).

The results for the periodic surface potential are in agreement with those obtained for the continuous Schrödinger operator in dimensions 2 and 3 with the potential of the form of the sum of the point potentials whose centers are periodic on the line $x_{1}=0$, and on the plane $x_{1}=0[3,11,12]$.

\section{References}

[1] Cornfeld, I. P., Fomin, S. V., Sinai, Ya. G. Ergodic Theory. Springer-Verlag, New York, 1982

[2] Figotin, A. L.; Pastur, L. A. An exactly solvable model of a multidimensional incommensurate structure. Comm. Math. Phys. 95 (1984), no. 4, 401-425

[3] Grossmann, A., Hoegh-Krohn, R., Mebkhout, M. The one particle theory of periodic point interactions. Polymers, monomolecular layers, and crystals Comm. Math. Phys. 77 (1980) 87-110.

[4] Jakšić, V., Last, Y. Spectral structure of Anderson type Hamiltonians Invent. Math. 141 (2000) 561-577 
[5] Jakšić, V., Last, Y. Corrugated surfaces and a.c. spectrum. Rev. Math. Phys. 12 (2000) 1465-1503

[6] Jakšić, V., Molchanov, S. On the spectrum of the surface Maryland model. Lett. Math. Phys. 45 (1998) 189-193

[7] Jakšić, V. Molchanov, S. On the surface spectrum in dimension two. Helv. Phys. Acta 71 (1998) 629-657

[8] Jakšić, V., Molchanov, S. Localization of surface spectra. Comm. Math. Phys. 208 (1999) 153-172

[9] Jakšić, V., Molchanov, S. Wave operators for the surface Maryland model. J. Math. Phys. 41 (2000) 4452-4463

[10] Jaksic, V., Molchanov, S., Pastur, L. On the propagation properties of surface waves In: IMA Vol. Math. Appl.96, Springer, New York, 1998, pp. 145-154

[11] Karpeshina, Yu. E. The spectrum and eigenfunctions of the Schrödinger operator in a three-dimensional space with point-like potential of the homogeneous two-dimensional lattice type. (Russian) Teoret. Mat. Fiz. 57 (1983) 414-423

[12] Karpeshina, Yu. E. An eigenfunction expansion theorem for the Schrödinger operator with a homogeneous simple two-dimensional lattice of potentials of zero radius in a three-dimensional space. (Russian) Vestnik Leningrad. Univ. Mat. Mekh. Astronom. 1984, vyp. 1, 11-17.

[13] Khoruzhenko, B., Pastur, L. Localisation of surface states: an explicitly solvable model Physics Reports 288 (1997) 109-125.

[14] Pastur, L, Surface waves: propagation and localisation In: Journées "Equations aux Dérivées Partielles", (Saint-Jean-de-Monts, 1995), Exp. No. VI, 12 pp.

[15] Pastur, L., Figotin A. Spectra of Random and Almost Periodic Operators Spriger Verlag, Berlin-Heidelberg, 1992

[16] Pearson, D. B. Quantum Scattering and Spectral Theory. Academic Press, London, 1988

[17] Reed, M., Simon, B. Methods of Modern Mathematical Physics. III. Scattering Theory. Academic Press, New York-London, 1979

[18] Simon, B. Quantum Mechanics for Hamiltonians Defined as Quadratic Forms. Princeton University Press, Princeton, N. J., 1971. xv+244 pp.

[19] Simon, B. Almost periodic Schrödinger operators. IV. The Maryland model. Ann. Physics 159 (1985) 157-183 
Centre de Physique Théorique, CASE 907,

13288 LUMINY

FRANCE

bento@cpt.univ-mrs.fr

briet@cpt.univ-mrs.fr

pastur@cpt.univ-mrs.fr 